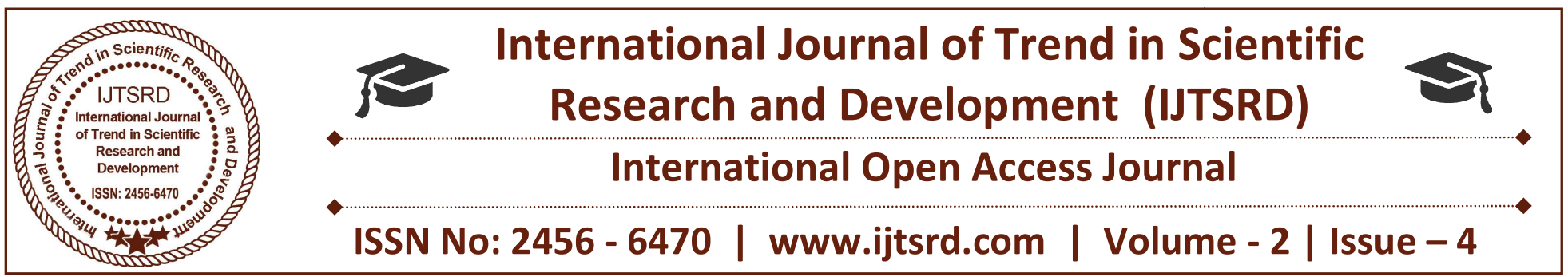

\title{
Application of Convolution Theorem
}

\author{
Dr. Dinesh Verma \\ Associate Professor, Yogananda College of Engineering \& Technology, Jammu, India
}

\begin{abstract}
Generally it has been noticed that differential equation is solved typically. The Laplace transformation makes it easy to solve. The Laplace transformation is applied in different areas of science, engineering and technology. The Laplace transformation is applicable in so many fields. Laplace transformation is used in solving the time domain function by converting it into frequency domain. Laplace transformation makes it easier to solve the problems in engineering applications and makes differential equations simple to solve. In this paper we will discuss how to follow convolution theorem holds the Commutative property, Associative Property and Distributive Property.
\end{abstract}

Keywords: Laplace transformation, Inverse Laplace transformation, Convolution theorem

\section{INTRODUCTION:}

Laplace transformation is a mathematical tool which is used in the solving of differential equations by converting it from one form into another form. Generally it is effective in solving linear differential equation either ordinary or partial. It reduces an ordinary differential equation into algebraic equation. Ordinary linear differential equation with constant coefficient and variable coefficient can be easily solved by the Laplace transformation method without finding the generally solution and the arbitrary constant. It is used in solving physical problems. this involving integral and ordinary differential equation with constant and variable coefficient.

It is also used to convert the signal system in frequency domain for solving it on a simple and easy way. It has some applications in nearly all engineering disciplines, like System Modeling, Analysis of Electrical Circuit, Digital Signal Processing, Nuclear
Physics, Process Controls, Applications in Probability, Applications in Physics, Applications in Power Systems Load Frequency Control etc.

\section{DEFINITION}

Let $\mathrm{F}(\mathrm{t})$ is a well defined function of $\mathrm{t}$ for all $\mathrm{t} \geq 0$. The Laplace transformation of $\mathrm{F}(\mathrm{t})$, denoted by $\mathrm{f}(p)$ or $\mathrm{L}\{\mathrm{F}(\mathrm{t})\}$, is defined as

$$
\mathrm{L}\{\mathrm{F}(\mathrm{t})\}=\int_{0}^{\infty} e^{-p t} F(t) d t=f(p)
$$

Provided that the integral exists, i.e. convergent. If the integral is convergent for some value of $p$, then the Laplace transformation of $F(t)$ exists otherwise not. Where $p$ the parameter which may be real or complex number and $\mathrm{L}$ is is the Laplace transformation operator.

The Laplace transformation of $\mathrm{F}$ (t) i.e. $\int_{0}^{\infty} e^{-p t} F(t) d t$ exists for $p>\mathrm{a}$, if

$\mathrm{F}(\mathrm{t})$ is continuous andlim ${ }_{n \rightarrow \infty}\left\{e^{-a t} F(t)\right\}$ is finite. It should however, be keep in mind that above condition are sufficient and not necessary.

\section{Inverse Laplace Transformation}

\section{Definition:}

If be the Laplace Transformation of a function $\mathrm{F}(\mathrm{t})$, then $\mathrm{F}(\mathrm{t})$ is called the Inverse Laplace transformation of the function $\mathrm{f}(\mathrm{p})$ and is written as $F(t)=L^{-1}\{f(p)\}$, Where $L^{-1}$ is called the

inverse Laplace transformation.

General Property of inverse Laplace transformation, 
(1) Linearty Property:

If $c_{1}$ and $c_{2}$ are constants and if

$L^{-1}\{f(p)\}=F(t) \operatorname{and} L\{G(t)\}=g(p)$

then,

$$
\begin{aligned}
& L^{-1}\left[c_{1} f(p)+c_{2} g(p)\right] \\
& \quad=c_{1} L^{-1}[f(p)]+c_{2} L^{-1}[g(p)]
\end{aligned}
$$

(2)First Shifting Property:

if $L^{-1}\{f(p)\}=F(t)$, then

$L^{-1}\{f(p-a)\}=e^{a t} F(t)$,

(3)Change of Scale Property:

if $L^{-1}\{f(p)\}=F(t)$, then

$L^{-1}\{f(a p)\}=\frac{1}{a} F\left(\frac{t}{a}\right)$,

(4)Inverse Laplace Transformation of

derivative:

if $L^{-1}\{f(p)\}=F(t)$, then

$L^{-1}\left[-\frac{d\{f(p)\}}{d p}\right]=t F(t)$

$L^{-1}\left[-\frac{d^{n}\{f(p)\}}{d p^{n}}\right]=(-1)^{n} t^{n} F(t)$,

whrer $n=123 \ldots \ldots \ldots$

(5)Inverse Laplace Transformation of

integral:

if $L^{-1}\{f(p)\}=F(t)$, then

$L^{-1}\left[\int_{p}^{\infty} f(p) d p\right]=\frac{F(t)}{t}$

(6)Inverse Laplace Transformation of

division by $p$ :

if $L^{-1}\{f(p)\}=F(t)$, then

$L^{-1}\left[\frac{F(p)}{p}\right]=\int_{0}^{t} f(u) d u$

The convolution of two given functions acting an essential role in a number of physical applications. It is usually convenient to determination a Laplace transformation into the product of two transformations when the inverse transform of both transforms are known. Convolution is used to get inverse Laplace transformation in solving differential equations and integral equations.

If $H_{1}(t)$ and $H_{2}(t)$ be two functions of class $A$

and if $h_{1}(s)=L\left\{H_{1}(t)\right\}, h_{2}(s)=L\left\{H_{2}(t)\right\}$.

Then the convolution of these two

functions $H_{1}(t)$ and $H_{2}(t)$,

$t>0$ is defined by the integral

$\left\{H_{1} * H_{2}\right\}(t)=\int_{0}^{t} H_{1}(t) H_{2}(t-y) d y$

$$
\text { or } \int_{0}^{t} H_{1}(t-y) H_{2}(y) d y
$$

Which of course exists if $H_{1}(t)$ and $H_{2}(t)$ are piecewise continuous the relation is called the convolution or falting of $H_{1}(t)$ and $H_{2}(t)$.

Proof of convolution theorem:

By the definition of Laplace Transformation $L\left(H_{1} *\right.$ $\left.H_{2}\right)(t)=\int_{0}^{\infty} e^{-p t}\left\{\left(H_{1} * H_{2}\right) t\right\} d t$

$$
=\int_{0}^{\infty} e^{-p t}\left[\int_{0}^{t} H_{1}(t) H_{2}(t-y) d y\right] d t
$$

Where the double integral is taken over the infinite region in the first quadrant deceitful linking the limity $=0$ to $y=t$.

Now order of integral are changing

$$
\begin{gathered}
\int_{0}^{\infty} e^{-p t} H_{1}(y) d y \int_{y}^{\infty} e^{-p(t-y)} H_{2}(t-y) d t \\
t-y=u \Rightarrow d t=d u
\end{gathered}
$$

when the limit of $t$ is $y$ then the limit of $u$ is 0 and when the limit of $t$ is $\infty$ then the limit of $u$ is $\infty$.

Now from above

$$
\begin{gathered}
\int_{0}^{\infty} e^{-p t} H_{1}(y) d y \int_{y}^{\infty} e^{-p u} H_{2}(u) d u \\
h_{1}(p) h_{1}(p)
\end{gathered}
$$


International Journal of Trend in Scientific Research and Development (IJTSRD) ISSN: 2456-6470

Hence

$$
L\left(H_{1} * H_{2}\right)(t)=h_{1}(p) h_{1}(p)
$$

Properties of convolution theorem: convolution theorem holds the following Properties:

(1) Commutative Property: property states that there is no alter in result from side to side the numbers in an appearance are exchange. Commutative property holds for addition and multiplication but not for subtraction and division.

Addition $\quad H_{1}+H_{2}=H_{2}+H_{1}$

Subtration $\quad H_{1}-H_{2} \neq H_{2}-H_{1}$

Multiplecation $H_{1} * H_{2}=H_{2} * H_{1}$

Division $\quad H_{1} \div H_{2} \neq H_{2} \div H_{1}$

Now we will prove that the Commutative

Property for multiplecation

$\operatorname{Proof}(1)$ :

By the definition of convolution theorem

$$
\begin{array}{r}
H_{1} * H_{2}=\int_{0}^{t} H_{1}(t) H_{2}(t-y) d y \\
\text { Let } t-y=u \Rightarrow d t=-d u,
\end{array}
$$

when the limit of $y$ is 0 then $u$ is $y$

and when $y$ is then $u$ is 0 .

$$
\begin{aligned}
& H_{1} * H_{2}=\int_{0}^{t} H_{1}(t-u) H_{2}(u) d u \\
& H_{1} * H_{2}=\int_{0}^{t} H_{1}(t-u) H_{2}(u) d u \\
& H_{1} * H_{2}=\int_{0}^{t} H_{2}(u) H_{1}(t-u) d u \\
& H_{1} * H_{2}=H_{2} * H_{1}
\end{aligned}
$$

The Convolution of $H_{1}$ and $H_{2}$ follow the commutative property.

(2) Associative Property: Associative Property states that the order of grouping the numbers does not matter. This law holds for addition and multiplication but not for subtraction and division.

$$
\text { Addition } H_{1}+\left(H_{2}+H_{3}\right)=\left(H_{1}+H_{2}\right)+H_{3}
$$

Hence, $H_{1} * H_{4}=\int_{0}^{t} H_{1}(z) H_{4}(t-z) d z$

Subtration $H_{1}-\left(H_{2}-H_{3}\right)=\left(H_{1}-H_{2}\right)-H_{3}$ Multiplecation $H_{1} *\left(H_{2} * H_{3}\right)=\left(H_{1} * H_{2}\right) * H_{3}$

$$
\text { Division } H_{1} \div\left(H_{2} \div H_{3}\right)=\left(H_{1} \div H_{2}\right) \div H_{3}
$$

Now we will see that how to convolution theorem follow the Associative property for multiplication

$$
\begin{gathered}
H_{1} *\left(H_{2} * H_{3}\right)=\left(H_{1} * H_{2}\right) * H_{3} \\
\text { let } H_{2} * H_{3}=H_{4}
\end{gathered}
$$

$$
\text { Now, } H_{4}=H_{2} * H_{3}=\int_{0}^{t} H_{2}(y) H_{3}(t-y) d y
$$

From above the commutative property

$$
H_{1} * H_{2}=H_{2} * H_{1}
$$

Therefore $\mathrm{H}_{2} * \mathrm{H}_{3}=\mathrm{H}_{3} * \mathrm{H}_{2}$

$$
\text { So, } H_{3} * H_{2}=\int_{0}^{t} H_{3}(y) H_{2}(t-y) d y
$$

$$
H_{1} * H_{4}=\int_{0}^{t} H_{1}(z)\left[\int_{0}^{t-z} H_{3}(y) H_{2}(t-z\right.
$$

$$
-y) d y] d z
$$

Change of order of integration

$$
\begin{aligned}
& H_{1} * H_{4}=\int_{0}^{t} H_{3}(z)\left[\int_{0}^{t-z} H_{1}(y) H_{2}(t-z-\right. \\
& y) d z] d y=H_{3}\left(H_{1} * H_{2}\right) \\
& \text { Hence, } H_{1} *\left(H_{2} * H_{3}\right)=\left(H_{1} * H_{2}\right) * H_{3}
\end{aligned}
$$

Distributive Property: The property with respect to addition is used to eliminate the bracket in an expression. The distributive property states that each term inside the bracket should be multiplied with the term outside. The property is very useful while simplifying the expressions and solving the complicated equations.

Distributive property over addition

$H_{1} *\left(H_{2}+H_{3}\right)=H_{1} * H_{2}+H_{1} * H_{3}$

Here the terms which are inside the bracket $\left(H_{1}\right.$ and $\left.H_{2}\right)$ are multiplied with the external terms (while is $H_{1}$ ). 
Now we will see that how to convolution theorem follow the Distributive property for multiplication

$H_{1} *\left(H_{2}+H_{3}\right)=\int_{0}^{t} H_{1}(y)\left[H_{2}(t-y)+H_{2}(t-\right.$

$y)] d y$

$=\int_{0}^{t} H_{1}(y) H_{2}(t-y) d y+\int_{0}^{t} H_{1}(y) H_{2}(t-$

$y) d y$

$H_{1} * H_{2}+H_{1} * H_{3}$

Hence, $H_{1} *\left(H_{2}+H_{3}\right)=H_{1} * H_{2}+H_{1} * H_{3}$

\section{CONCLUSION:}

In this paper we have discussed the Applications of convolution theorem of Laplace transformation i.e. how to follow the convolution theorem holds the Commutative property, Associative Property and Distributive Property. The primary use of Laplace transformation is converting a time domain functions into frequency domain function. Here, Some Property of inverse Laplace transformations like, linearity property, First shifting property, Change of scale property, Inverse Laplace transformation of derivative etc. has been discussed.

\section{REFERENCES:}

1. B. V. Ramana, Higher Engineering Mathematics.

2. Dr. B. S. Grewal, Higher Engineering Mathematics.

3. Dr. S. K. Pundir, Engineering Mathematics with gate tutor.

4. Erwin Kreyszig, Advanced Engineering Mathematics, Wiley, 1998.

5. J.L. Schiff, The Laplace Transform: Theory and Applications, Springer Science and Business Media (1999).

6. Advanced engineering mathematics seventh edition, peter v. Oneil.

7. H. K. Dass, 'Higher engineering Mathematics",

S. Chand and company limited, New Delhi 